Delft University of Technology

\title{
Comparison of three control structures for inducing higher-order sliding modes
}

Wang, Xuerui; Van Kampen, Erik Jan; Chu, Qiping

DOI

10.23919/ECC.2019.8795872

Publication date

2019

Document Version

Final published version

Published in

2019 18th European Control Conference, ECC 2019

\section{Citation (APA)}

Wang, X., Van Kampen, E. J., \& Chu, Q. (2019). Comparison of three control structures for inducing higherorder sliding modes. In 2019 18th European Control Conference, ECC 2019 (pp. 4073-4080). [8795872] Institute of Electrical and Electronics Engineers (IEEE). https://doi.org/10.23919/ECC.2019.8795872

\section{Important note}

To cite this publication, please use the final published version (if applicable).

Please check the document version above.

\section{Copyright}

Other than for strictly personal use, it is not permitted to download, forward or distribute the text or part of it, without the consent of the author(s) and/or copyright holder(s), unless the work is under an open content license such as Creative Commons.

\section{Takedown policy}

Please contact us and provide details if you believe this document breaches copyrights.

We will remove access to the work immediately and investigate your claim. 
Green Open Access added to TU Delft Institutional Repository

'You share, we take care!' - Taverne project

https://www.openaccess.nl/en/you-share-we-take-care

Otherwise as indicated in the copyright section: the publisher is the copyright holder of this work and the author uses the Dutch legislation to make this work public. 


\title{
Comparison of Three Control Structures for Inducing Higher-Order Sliding Modes
}

\author{
Xuerui Wang ${ }^{1}$, Erik-Jan van Kampen ${ }^{2}$, and Qiping $\mathrm{Chu}^{3}$
}

\begin{abstract}
For mitigating the chattering effect in the sliding mode control (SMC), many adaption mechanisms have been proposed to reduce the switching gains. However, less attention is paid to the control structure, which influences the resulting uncertainty term and determines the minimum possible gains. This paper compares three control structures for inducing higher-order sliding modes in finite time: nonlinear dynamic inversion (NDI) based SMC, higher-order sliding mode control (HOSMC) with artificially increased relative degree, and the recently proposed incremental nonlinear dynamic inversion (INDI) based SMC. The latter two control structures have reduced model dependency as compared to NDI-SMC. Moreover, their nominal control increments are found to be approximately equivalent if the sampling interval is sufficiently small and if their gains satisfy certain conditions. Under the same circumstances, the norm value of the resulting uncertainty using INDI-SMC is several orders of magnitude smaller than those using other control structures. For maintaining the sliding modes, the minimum possible gains required by HOSMC approximately equal those needed by INDI-SMC divided by the sampling interval. Nevertheless, these two approaches have comparable chattering degrees, which are effectively reduced as compared to NDI-SMC. The analytical results are verified by numerical simulations.
\end{abstract}

\section{INTRODUCTION}

Featured by its invariance to the matched uncertainty and its finite time convergence property, Sliding Mode Control (SMC) is a useful approach for stabilizing perturbed systems. However, many SMC methods suffer from the chattering phenomenon, which is the high frequency switching in the control signal. Although continuous approximations of the signum function and a class of higher order sliding mode control (HOSMC) techniques can mitigate the chattering effect, it is shown in [1] that none of these methods can totally eliminate chattering.

In view of the fact that the chattering amplitude is proportional to the discontinuous switching gain [2], a recent research focus in the SMC community is on adapting the switching gains to their minimum possible values whilst maintaining the sliding modes and the finite-time convergence property [1], [2], [3], [4]. An adaption mechanism for the super-twisting algorithm based on direct measurements of the equivalent control is presented in [2]. A dual-layer nested

\footnotetext{
${ }^{1}$ Xuerui Wang is with Faculty of Aerospace Engineering, Delft University of Technology; Kluyverweg 1, 2629HS, Delft, The Netherlands. X.Wang-6etudelft.nl

${ }^{2}$ Erik-Jan van Kampen is with Faculty of Aerospace Engineering, Delft University of Technology; Kluyverweg 1, 2629HS, Delft, The Netherlands. E.vanKampen@tudelft.nl

${ }^{3}$ Qiping Chu is with Faculty of Aerospace Engineering, Delft University of Technology; Kluyverweg 1, 2629HS, Delft, The Netherlands. Q.P.Chu@tudelft.nI
}

adaptive method is proposed in [4] and further developed in [1], [3], which can be applied to super-twisting, conventional (first-order), and higher-order sliding mode schemes. These methods are derived for the sliding variable dynamics, which contain a lumped uncertainty term and decoupled control inputs. However, the real system dynamics usually have coupled control inputs, and are perturbed by both additive and multiplicative uncertainties. Converting the real coupled system dynamics to the decoupled sliding variable dynamics is an important intermediate step which has been overlooked in the literature. This issue is influential because different control structures used for this conversion will result in different closed-loop uncertainty terms which are crucial to the minimum possible values of the sliding mode control/observer gains.

Feedback linearization, also known as Nonlinear Dynamic Inversion (NDI) in the aerospace community [5], [6], [7] is a widely used control structure for solving nonlinear system sliding mode control problems. A recent review of NDI based SMC with various sliding orders and virtual control designs can be found in [8]. It is also shown in [8] that SMC which contains a model-based estimation of the equivalent control [1], [2] is essentially NDI based. However, the NDI control structure has a contradiction between the reduction of uncertainty and model dependency. To be specific, reducing the model dependency of NDI is beneficial for complex system (such as an airplane) control implementations, but would increase the norm of the remaining uncertainties in the sliding variable dynamics, which eventually requires higher sliding mode control/observer gain for perturbation compensations.

In order to solve this contradiction, recent research proposes to use the control structure of Incremental Nonlinear Dynamic Inversion (INDI) for inducing sliding modes [8]. INDI is a so-called, sensor-based control approach developed in the aerospace community, which designs the control increment $\Delta \boldsymbol{u}$ in one sampling interval $\Delta t$ [5]. The idea of INDI can be traced back to the late nineties, when it was found in [9] that the feedback of angular acceleration and control surface position can enhance the robustness of NDI to model uncertainties. Inheriting this idea, INDI was first proposed in [6], and has shown promising effectiveness on faulttolerant flight control [7], helicopter control [10], flexible aircraft control [11], and high-speed control of a damaged quadrotor with complete loss of a single rotor [12], in the past decade. A recent research generalized INDI for generic nonlinear uncertain systems with arbitrary relative degree, without using the time-scale separation assumption [5]. It 
is found in [5], that part of the system dynamics and external disturbances are contained in the output derivatives and control inputs at the previous time step. Therefore, feeding back their latest samples/estimations can improve the robustness of NDI to regular perturbations while reducing its model dependency. This analytical conclusion is verified by real-world passenger airplane flight tests in [13], [14]. The sensor-based control idea of INDI is also shared by [15], [16], where the signals at the previous time step are used to estimate disturbance. Different from [15], [16], the only model information required by INDI is the estimation of the control effectiveness matrix. By virtue of the sensorbased nature of INDI, SMC using the control structure of INDI instead of NDI can simultaneously reduce the control model dependency and uncertainties remaining in the sliding variable dynamics [8], which is beneficial for chattering reduction.

Another strategy for chattering mitigation is artificially increasing the input-output relative degree, and designing the higher-order derivatives of the control input [17], [18]. The actual control input is then integrated from the designed control derivatives. Because of this integration process, the high frequency chattering can be smoothened. Although this strategy is widely used, the property of the resulting uncertainty term and its influences on the minimum possible SMC gains remain unclear in the literature. In this paper, particular interest is paid to HOSMC which artificially increases the relative degree by one order (designs $\dot{\boldsymbol{u}}$ ). Since $\dot{\boldsymbol{u}}$ approximately equals $\Delta \boldsymbol{u} / \Delta t$ when $\Delta t$ is sufficiently small, an interesting research question emerges: is there any relationship between the INDI-SMC and HOSMC with artificially increased relative degree, although these two methods originate from completely different ideas?

The main contributions of this paper are the comparisons among three different control structures that can be used for inducing higher-order sliding modes. These three control structures are NDI-SMC and HOSMC with artificially increased relative degree developed in the SMC community, and the INDI-SMC recently proposed in the aerospace community. The comparisons are mainly in three aspects: 1) Model dependency and the required signals for implementation; 2) The relationships of the nominal controls; 3) The properties of the resulting uncertainty terms and their influences on the minimum possible values of the sliding mode control/observer gains.

This paper is structured as follows: The three control structures will be presented in Sec. II, analytically compared in Sec. III, and numerically compared in Sec. IV. Main conclusions are drawn in Sec. V.

\section{Three Control Structures For INDUCing HIGHER-ORDER SLIDING MODES}

Consider a multi-input/multi-output nonlinear controlaffine system

$$
\dot{\boldsymbol{x}}=\boldsymbol{f}(\boldsymbol{x})+\boldsymbol{G}(\boldsymbol{x}) \boldsymbol{u}+\boldsymbol{d}_{x}, \quad \boldsymbol{y}=\boldsymbol{h}(\boldsymbol{x})
$$

where $f: \mathbb{R}^{n} \rightarrow \mathbb{R}^{n}$ and $\boldsymbol{h}: \mathbb{R}^{n} \rightarrow \mathbb{R}^{m}$ are smooth vector fields. $\boldsymbol{G}$ is a smooth function mapping $\mathbb{R}^{n} \rightarrow$ $\mathbb{R}^{n \times m}$, whose columns are smooth vector fields. $\boldsymbol{d}_{x} \in \mathbb{R}^{n}$ represents external disturbances. Boldfaces indicate vectors and matrices.

Assumption 1: The vector relative degree of $\boldsymbol{y}$ with respect to $\boldsymbol{u}$ denoted as $\boldsymbol{r}=\left[r_{1}, r_{2}, \ldots, r_{m}\right]^{T}$ is constant and known, and the corresponding internal dynamics are stable.

By differentiating the output, the input-output mapping of the system is given by

$$
\boldsymbol{y}^{(\boldsymbol{r})}=\boldsymbol{\alpha}(\boldsymbol{x})+\mathcal{B}(\boldsymbol{x}) \boldsymbol{u}+\boldsymbol{d}
$$

where $\boldsymbol{y}^{(\boldsymbol{r})}=\left[y_{1}^{\left(r_{1}\right)}, \ldots, y_{m}^{\left(r_{m}\right)}\right]^{T}, \mathcal{B}(\boldsymbol{x}) \in \mathbb{R}^{m \times m}$, $\mathcal{B}_{i j}=\mathcal{L}_{g_{j}} \mathcal{L}_{f}^{r_{i}-1} h_{i}, \boldsymbol{\alpha}(\boldsymbol{x})=\left[\mathcal{L}_{f}^{r_{1}} h_{1}, \ldots, \mathcal{L}_{f}^{r_{m}} h_{m}\right]^{T}, \boldsymbol{d}=$ $\left[\mathcal{L}_{d_{x}}^{r_{1}} h_{1}, \ldots, \mathcal{L}_{d_{x}}^{r_{m}} h_{m}\right]^{T}$, with $\mathcal{L}_{f}^{r_{i}} h_{i}, \mathcal{L}_{d_{x}}^{r_{i}} h_{i}, \mathcal{L}_{g_{j}} \mathcal{L}_{f}^{r_{i}-1} h_{i}$ the corresponding Lie derivatives. The control effectiveness matrix $\mathcal{B}(\boldsymbol{x})$ is nonsingular under Assumption 1.

The control objective is to make the output $\boldsymbol{y}$ track a reference signal $\boldsymbol{y}_{c}(t)=\left[y_{c_{1}}(t), \ldots, y_{c_{m}}(t)\right]^{T}$. Assume $y_{c_{i}}(t), i=1, \ldots, m$, and its derivatives up to $y_{c_{i}}^{\left(r_{i}+1\right)}(t)$ are bounded for all $t$. Choose the sliding variable as $\boldsymbol{\sigma}=\boldsymbol{y}-\boldsymbol{y}_{c}$.

Definition 1: [17], [18] Consider the nonlinear system given by Eq. (1) and the sliding variable $\sigma$, assume the time derivatives of $\sigma_{i}, \dot{\sigma}_{i}, \ldots, \sigma_{i}{ }^{\left(r_{i}-1\right)}$ are continuous functions for all $i=1, \ldots, m$. The manifold defined as

$\mathcal{S}^{r}=\left\{\boldsymbol{x} \mid \sigma_{i}(\boldsymbol{x})=\dot{\sigma}_{i}(\boldsymbol{x}) \ldots=\sigma_{i}{ }^{\left(r_{i}-1\right)}(\boldsymbol{x})=0, i=1, \ldots m\right\}$

is called the " $r^{t h}$-order sliding set" [18], [19]. If this sliding set is non empty and locally an integral set in the Filippov sense [20], then the motion on $\mathcal{S}^{r}$ is called the " $\boldsymbol{r}^{t h}$-order sliding mode" with respect to the sliding variable $\sigma$.

In the following subsections, three control structures for inducing the $\boldsymbol{r}^{\text {th }}$-order sliding mode will be presented.

\section{A. Nonlinear Dynamic Inversion based Control}

The estimated system dynamics $\boldsymbol{y}^{(\boldsymbol{r})}=\overline{\boldsymbol{\alpha}}(\boldsymbol{x})+\overline{\mathcal{B}}(\boldsymbol{x}) \boldsymbol{u}$ are used for control design, which bring both additive uncertainties $\boldsymbol{\alpha}-\overline{\boldsymbol{\alpha}}+\boldsymbol{d}$, and multiplicative uncertainties $\mathcal{B}-\overline{\mathcal{B}}$. For inducing the $\boldsymbol{r}^{t h}$-order sliding mode, the Nonlinear Dynamic Inversion (NDI) control structure is adopted in [18], [21] as:

$$
\boldsymbol{u}_{\text {ndi-s }}=\overline{\mathcal{B}}^{-1}(\boldsymbol{x})\left(\boldsymbol{\nu}_{n}+\boldsymbol{\nu}_{s}+\boldsymbol{y}_{c}^{(\boldsymbol{r})}-\overline{\boldsymbol{\alpha}}(\boldsymbol{x})\right)
$$

where $\boldsymbol{y}_{c}^{(\boldsymbol{r})}=\left[y_{c_{1}}^{r_{1}}, y_{c_{2}}^{r_{2}}, \ldots, y_{c_{m}}^{r_{m}}\right]^{T}$. $\boldsymbol{\nu}_{n}$ and $\boldsymbol{\nu}_{s}$ are two virtual control terms. This control structure will be abbreviated as NDI-SMC in the subsequent context. Using Eq. (4), the dynamics of $\boldsymbol{\sigma}$ are

$$
\begin{aligned}
\boldsymbol{\sigma}^{(\boldsymbol{r})} & =\boldsymbol{y}^{(\boldsymbol{r})}-\boldsymbol{y}_{c}^{(\boldsymbol{r})}=\overline{\boldsymbol{\alpha}}(\boldsymbol{x})+\overline{\mathcal{B}}(\boldsymbol{x}) \boldsymbol{u}_{\mathrm{ndi}-s}+\varepsilon_{\mathrm{ndi}-s}-\boldsymbol{y}_{c}^{(\boldsymbol{r})} \\
& =\boldsymbol{\nu}_{n}+\boldsymbol{\nu}_{s}+\boldsymbol{\varepsilon}_{\mathrm{ndi}-s}
\end{aligned}
$$

where

$$
\begin{aligned}
\varepsilon_{\mathrm{ndi}-s} & =(\boldsymbol{\alpha}-\overline{\boldsymbol{\alpha}})+\left(\mathcal{B} \overline{\mathcal{B}}^{-1}-\boldsymbol{I}\right)\left(\boldsymbol{\nu}_{n}+\boldsymbol{\nu}_{s}+\boldsymbol{y}_{c}^{(\boldsymbol{r})}-\overline{\boldsymbol{\alpha}}\right) \\
& =(\boldsymbol{\alpha}-\overline{\boldsymbol{\alpha}})+(\mathcal{B}-\overline{\mathcal{B}}) \boldsymbol{u}_{\mathrm{ndi}-s}+\boldsymbol{d}
\end{aligned}
$$

$\varepsilon_{\text {ndi-s }}$ in Eq. (6) is the closed-loop uncertainty term caused by model uncertainties, external disturbances, actuator faults, 
structural damage, etc [8]. If $\nu_{s}$ can compensate for $\varepsilon_{\text {ndi-s }}$, and if the nominal virtual control $\boldsymbol{\nu}_{n}$ can stabilize the unperturbed $\boldsymbol{r}^{t h}$-order integrator chains [18], [22], then the $\boldsymbol{r}^{t h}$-order sliding mode can be established by Eq. (4).

\section{B. Incremental Sliding Mode Control}

Incremental Sliding Mode Control (INDI-SMC) is a recently proposed control framework [8] which can simultaneously reduce the model dependency and the minimum possible switching gains of NDI-SMC. Denote the sampling interval as $\Delta t$. To begin with, the incremental dynamic equation is derived by taking the first-order Taylor series expansion of Eq. (2) around the condition at $t-\Delta t$ (denoted by the subscript 0 ) as

$$
\begin{aligned}
\boldsymbol{y}^{(\boldsymbol{r})=} & \boldsymbol{y}_{0}^{(\boldsymbol{r})}+\mathcal{B}\left(\boldsymbol{x}_{0}\right) \Delta \boldsymbol{u}+\Delta \boldsymbol{d} \\
& +\left.\frac{\partial[\boldsymbol{\alpha}(\boldsymbol{x})+\mathcal{B}(\boldsymbol{x}) \boldsymbol{u}]}{\partial \boldsymbol{x}}\right|_{0} \Delta \boldsymbol{x}+\mathcal{O}\left(\Delta \boldsymbol{x}^{2}\right)
\end{aligned}
$$

where $\Delta x=x-x_{0}, \Delta u=u-u_{0}, \Delta d=d-d_{0}$ respectively denote the variations of states, control inputs and external disturbances in one incremental time step. Based on Eq. (7), the INDI-SMC framework is proposed as [8]

$$
\Delta \boldsymbol{u}_{\text {indi-s }}=\overline{\mathcal{B}}^{-1}\left(\boldsymbol{x}_{0}\right)\left(\boldsymbol{\nu}_{n}+\boldsymbol{\nu}_{s}+\boldsymbol{y}_{c}^{(\boldsymbol{r})}-\boldsymbol{y}_{0}^{(\boldsymbol{r})}\right)
$$

The total control command vector for the actuators is $\boldsymbol{u}_{\text {indi- } s}=\boldsymbol{u}_{\text {indi- } s, 0}+\Delta \boldsymbol{u}_{\text {indi- } s}$.

Remark 1: $\boldsymbol{u}_{\text {indi-s, }, 0}$ denotes the latest sampled actuator position vector, instead of the control command given to the actuator at the previous time step. The flight tests in [23] shows that this sensing process can mitigate the influences of actuator dynamics. If actuator dynamics are not considered, $\boldsymbol{u}_{\text {indi-s }}=\sum \Delta \boldsymbol{u}_{\text {indi-s }}$, which is essentially a numerical integration process.

As compared to the NDI based control structure (Eq. (4)), INDI-SMC is independent of the model information $\overline{\boldsymbol{\alpha}}(\boldsymbol{x})$, which simplifies the implementation process. Only the estimation of the control effectiveness $(\overline{\mathcal{B}})$ is needed by INDISMC. The calculation of the partial derivatives in Eq. (7) is not needed for control implementation. It will be shown later that the multiplications of these partial derivatives with $\Delta \boldsymbol{x}$ are treated as perturbations in the closed-loop system. This approach also inherits the sensor-based nature of INDI, since the measurements/estimations of $\boldsymbol{y}_{0}^{(\boldsymbol{r})}$ and $\boldsymbol{u}_{\text {indi- } s, 0}$ are needed. For some physical systems, including aircraft, $\boldsymbol{y}_{0}^{(\boldsymbol{r})}$ and $\boldsymbol{u}_{\text {indi- } s, 0}$ can be directly measured [13], [14].

Finally, substituting Eq. (8) into Eq. (7), and using the definition of $\boldsymbol{\sigma}$, the resulting dynamics are

$$
\begin{aligned}
\boldsymbol{\sigma}^{(\boldsymbol{r})} & =\boldsymbol{y}^{(\boldsymbol{r})}-\boldsymbol{y}_{c}^{(\boldsymbol{r})}=\boldsymbol{y}_{0}^{(\boldsymbol{r})}+\overline{\mathcal{B}}\left(\boldsymbol{x}_{0}\right) \Delta \boldsymbol{u}_{\text {indi-s }}+\boldsymbol{\varepsilon}_{\text {indi-s }}-\boldsymbol{y}_{c}^{(\boldsymbol{r})} \\
& =\boldsymbol{\nu}_{n}+\boldsymbol{\nu}_{s}+\boldsymbol{\varepsilon}_{\text {indi-s }}
\end{aligned}
$$

where

$$
\begin{aligned}
\varepsilon_{\text {indi- } s}= & \left.\frac{\partial\left[\boldsymbol{\alpha}(\boldsymbol{x})+\mathcal{B}(\boldsymbol{x}) \boldsymbol{u}_{\text {indi-s }}\right]}{\partial \boldsymbol{x}}\right|_{0} \boldsymbol{\Delta} \boldsymbol{x}+\mathcal{O}\left(\Delta \boldsymbol{x}^{2}\right) \\
& +\left.(\mathcal{B}-\overline{\mathcal{B}})\right|_{0} \Delta \boldsymbol{u}_{\text {indi-s }}+\Delta \boldsymbol{d}
\end{aligned}
$$

in which $\left.(\mathcal{B}-\overline{\mathcal{B}})\right|_{0}$ denotes $\mathcal{B}\left(\boldsymbol{x}_{0}\right)-\overline{\mathcal{B}}\left(\boldsymbol{x}_{0}\right)$. Eq. (9) is in the same form as Eq. (5), thus the $\boldsymbol{r}^{\text {th }}$-order sliding mode can be achieved by properly designing $\boldsymbol{\nu}_{n}$ and $\boldsymbol{\nu}_{s}$. However, the NDI and INDI based control structures result in different closed-loop perturbation terms: $\varepsilon_{\text {ndi-s }}$ and $\varepsilon_{\text {indi-s }}$. The properties of these two terms, and their influences on the minimum possible gains will be elaborated in III-B and III-C.

\section{Higher-order Sliding Mode Control with Artificially In- creased Relative Degree}

Consider artificially increasing $r_{i}$ by one order for all $i=$ $1, \ldots, m$. Dynamically extend Eq. (2) as

$$
\boldsymbol{y}^{(\boldsymbol{r}+\mathbf{1})}=\frac{\partial(\boldsymbol{\alpha}(\boldsymbol{x})+\mathcal{B}(\boldsymbol{x}) \boldsymbol{u})}{\partial \boldsymbol{x}} \dot{\boldsymbol{x}}+\mathcal{B}(\boldsymbol{x}) \dot{\boldsymbol{u}}+\dot{\boldsymbol{d}}
$$

Design the control derivative in the structure of

$$
\dot{\boldsymbol{u}}_{\mathrm{ho-s}}=\overline{\mathcal{B}}^{-1}(\boldsymbol{x})\left(\boldsymbol{\nu}_{n}^{\prime}+\boldsymbol{\nu}_{s}^{\prime}+\boldsymbol{y}_{c}^{(\boldsymbol{r}+\mathbf{1})}\right)
$$

which is referred to as HOSMC with artificially increased $\boldsymbol{r}$. This control structure leads to

$$
\begin{aligned}
\boldsymbol{\sigma}^{(\boldsymbol{r}+\mathbf{1})} & =\boldsymbol{y}^{(\boldsymbol{r}+\mathbf{1})}-\boldsymbol{y}_{c}^{(\boldsymbol{r}+\mathbf{1})}=\overline{\mathcal{B}}(\boldsymbol{x}) \dot{\boldsymbol{u}}_{\mathrm{ho}-s}+\varepsilon_{\mathrm{ho}-s}-\boldsymbol{y}_{c}^{(\boldsymbol{r}+\mathbf{1})} \\
& =\boldsymbol{\nu}_{n}^{\prime}+\boldsymbol{\nu}_{s}^{\prime}+\varepsilon_{\mathrm{ho}-s}
\end{aligned}
$$

where

$$
\varepsilon_{\mathrm{ho}-s}=\frac{\partial\left(\boldsymbol{\alpha}(\boldsymbol{x})+\mathcal{B}(\boldsymbol{x}) \boldsymbol{u}_{\mathrm{ho}-s}\right)}{\partial \boldsymbol{x}} \dot{\boldsymbol{x}}+(\mathcal{B}-\overline{\mathcal{B}}) \dot{\boldsymbol{u}}_{\mathrm{ho}-s}+\dot{\boldsymbol{d}}
$$

It is noteworthy that the $\boldsymbol{y}_{c}^{(\boldsymbol{r}+\mathbf{1})}$ term is viewed as uncertainty by [24], and is not used in Eq. (12). It is suggested in this paper to include this term in Eq. (12) as a known term. In view of Eq. (13), the $\boldsymbol{r}+\mathbf{1}^{\text {th }}$ - order sliding mode can be induced if $\nu_{s}^{\prime}$ can compensate for $\varepsilon_{\text {ho-s }}$, and if the nominal virtual control $\boldsymbol{\nu}_{n}^{\prime}$ can achieve the stabilization of the $\boldsymbol{r}+\mathbf{1}^{\text {th }}$-order unperturbed integrator chains [18], [22].

The actual control is integrated from Eq. (12) and can be further derived as

$$
\begin{aligned}
\boldsymbol{u}_{\mathrm{ho}-s} & =\int_{0}^{t} \dot{\boldsymbol{u}}_{\mathrm{ho}-s}=\int_{0}^{t-\Delta t} \dot{\boldsymbol{u}}_{\mathrm{ho}-s}+\int_{t-\Delta t}^{t} \dot{\boldsymbol{u}}_{\mathrm{ho}-s} \\
& \approx \boldsymbol{u}_{\mathrm{ho}-s, 0}+\dot{\boldsymbol{u}}_{\mathrm{ho}-s}(t) \Delta t
\end{aligned}
$$

which means the control increment of $\boldsymbol{u}_{\text {ho-s }}$ in $\Delta t$ approximately equals

$$
\Delta \boldsymbol{u}_{\mathrm{ho}-s} \approx \dot{\boldsymbol{u}}_{\mathrm{ho}-s}(t) \Delta t=\overline{\mathcal{B}}^{-1}(\boldsymbol{x})\left(\boldsymbol{\nu}_{n}^{\prime}+\boldsymbol{\nu}_{s}^{\prime}+\boldsymbol{y}_{c}^{(\boldsymbol{r}+\mathbf{1})}\right) \Delta t
$$

The control increment of $\boldsymbol{u}_{\text {ho- } s}$ is derived in Eqs. (15) and (16) for the convenience of comparison with Eq. (8). As compared to $\Delta \boldsymbol{u}_{\text {indi-s }}$ in Eq. (8), $\boldsymbol{u}_{\text {ho-s }}$ is also independent of the model information $\overline{\boldsymbol{\alpha}}(\boldsymbol{x})$. However, the $\boldsymbol{y}_{c}^{(\boldsymbol{r}+\mathbf{1})}$ term used in Eq. (16) is not needed by INDI-SMC (Eq. (8)). Other similarities and differences between this method and INDI$\mathrm{SMC}$ will be further explored in Sec. III.

\section{ANALYTICAL COMPARISONS}

In this section, the three control structures presented in Sec. II will be analytically compared. The nominal control parts will be considered in III-A. The properties of the perturbation terms will be compared in III-B. The perturbation compensations and minimum possible gain requirements will be discussed in III-C. 


\section{A. Comparisons of the Nominal Control}

This subsection considers the nominal case where the perturbation terms in Eqs. $(5,9,13)$ all equal to zero. The virtual controls $\boldsymbol{\nu}_{s}$ and $\boldsymbol{\nu}_{s}^{\prime}$ for perturbation compensation are also zero. Under this circumstance, the closed-loop dynamics using NDI-SMC and INDI-SMC become $\boldsymbol{r}^{\text {th }}$ order integrator chains with the nominal virtual control $\boldsymbol{\nu}_{n}$ as an input, namely $\boldsymbol{\sigma}^{(\boldsymbol{r})}=\boldsymbol{\nu}_{n}$. Analogously, using the control structure of HOSMC with artificially increased $r$, $\boldsymbol{\sigma}^{(\boldsymbol{r}+\mathbf{1})}=\boldsymbol{\nu}_{n}^{\prime}$ according to Eq. (13). Three approaches of the nominal dynamics stabilization will be discussed.

1) Asymptotic Stabilization: It is classical to stabilize the unperturbed integrator chains asymptotically by linear virtual controls. For NDI and INDI based SMC, $\nu_{n}$ in Eqs. $(4,8)$ is consistently designed as:

$$
\boldsymbol{\nu}_{n}=-\boldsymbol{K}_{r-1} \boldsymbol{\sigma}^{(\boldsymbol{r}-\mathbf{1})}-\boldsymbol{K}_{r-2} \boldsymbol{\sigma}^{(\boldsymbol{r}-\mathbf{2})} \ldots-\boldsymbol{K}_{0} \boldsymbol{\sigma}
$$

where the positive definite diagonal gain matrices $\boldsymbol{K}_{r-1}=$ $\operatorname{diag}\left\{K_{r-1, i}\right\}, \ldots, \boldsymbol{K}_{0}=\operatorname{diag}\left\{K_{0, i}\right\}, \quad i=1, \ldots, m$ are designed such that the $\boldsymbol{r}^{t h}$ - order polynomials $p^{r_{i}}+$ $K_{r-1, i} p^{r_{i}-1}+K_{r-2, i} p^{r_{i}-2} \ldots+K_{0, i}$ are Hurwitz.

Analogously, for asymptotically stabilizing the $r+\mathbf{1}^{t h}$ order integrator chains, $\boldsymbol{\nu}_{n}^{\prime}$ in Eq. (16) is designed as:

$$
\boldsymbol{\nu}_{n}^{\prime}=-\boldsymbol{K}_{r}^{\prime} \boldsymbol{\sigma}^{(\boldsymbol{r})}-\boldsymbol{K}_{r-1}^{\prime} \boldsymbol{\sigma}^{(\boldsymbol{r}-\mathbf{1})}-\boldsymbol{K}_{r-2}^{\prime} \boldsymbol{\sigma}^{(\boldsymbol{r}-\mathbf{2})} \ldots-\boldsymbol{K}_{0}^{\prime} \boldsymbol{\sigma}
$$

where $\boldsymbol{K}_{r}^{\prime}=\operatorname{diag}\left\{K_{r, i}^{\prime}\right\}, \ldots, \boldsymbol{K}_{0}^{\prime}=\operatorname{diag}\left\{K_{0, i}^{\prime}\right\}, i=1, \ldots, m$ are designed such that the $\boldsymbol{r}+\mathbf{1}^{t h}$ - order polynomials $p^{r_{i}+1}+$ $K_{r_{i}}^{\prime} p^{r_{i}}+K_{r-1, i}^{\prime} p^{r_{i}-1} \ldots+K_{0, i}^{\prime}$ are Hurwitz.

Using the definition of the sliding variable, $\sigma^{(\boldsymbol{r})}=$ $\boldsymbol{y}^{(\boldsymbol{r})}-\boldsymbol{y}_{c}^{(\boldsymbol{r})}$ in Eq. (18). Therefore, the nominal control part $\overline{\mathcal{B}}^{-1}\left(\boldsymbol{x}_{0}\right)\left(\boldsymbol{\nu}_{n}+\boldsymbol{y}_{c}^{(\boldsymbol{r})}-\boldsymbol{y}_{0}^{(\boldsymbol{r})}\right)$ in Eq. (8) approximately equals the nominal control increment $\overline{\mathcal{B}}^{-1}(\boldsymbol{x}) \boldsymbol{\nu}_{n}^{\prime} \Delta t$ in Eq. (16) if
1) $\overline{\mathcal{B}}^{-1}\left(\boldsymbol{x}_{0}\right) \approx \overline{\mathcal{B}}^{-1}(\boldsymbol{x})$
2) $\boldsymbol{y}^{(\boldsymbol{r})} \approx \boldsymbol{y}_{0}^{(\boldsymbol{r})}$
3) $\boldsymbol{K}_{r}^{\prime}=\frac{\boldsymbol{I}_{m}}{\Delta t}, \boldsymbol{K}_{r-1}^{\prime}=\frac{\boldsymbol{K}_{r-1}}{\Delta t}, \ldots, \boldsymbol{K}_{0}^{\prime}=\frac{\boldsymbol{K}_{0}}{\Delta t}$

where $\boldsymbol{I}_{m}$ is an $m$ dimensional identity matrix. Using the continuity of $\boldsymbol{x}, \lim _{\Delta t \rightarrow 0}\left\|\boldsymbol{x}-\boldsymbol{x}_{0}\right\|=0$. Therefore, the first condition can be satisfied when $\Delta t$ is sufficiently small. $\boldsymbol{y}_{0}^{(\boldsymbol{r})}$ used by Eq. (8) represents the value of $\boldsymbol{y}^{(\boldsymbol{r})}$ at the previous time step, which can be directly measured [5], [14] or estimated [13], [23]. For HOSMC, $\boldsymbol{y}^{(\boldsymbol{r})}$ is normally numerically differentiated from $\boldsymbol{y}$ using, for example, sliding mode differentiator [17], [24]. Although based on different ideas, $\boldsymbol{y}^{(\boldsymbol{r})}$ and $\boldsymbol{y}_{0}^{(\boldsymbol{r})}$ become close under sufficiently small $\Delta t$. The last gain condition can be derived by comparing Eq. (8) with Eq. (16), and comparing Eq. (17) with Eq. (18).

2) Finite Time Stabilization: The unperturbed integrator chains can also be stabilized in finite time using nonlinear continuous virtual controls.

Proposition 1: Consider the unperturbed integrator chain set $\boldsymbol{\sigma}^{(\boldsymbol{r})}=\boldsymbol{\nu}_{n}$, there exists $\epsilon_{i} \in(0,1), i=1, \ldots, m$, such that for every $\alpha_{i} \in\left(1-\epsilon_{i}, 1\right)$, the $\boldsymbol{r}^{t h}$ - order sliding mode is established in finite time by

$$
\begin{aligned}
\boldsymbol{\nu}_{n}= & {\left[\nu_{n, 1}, \nu_{n, 2}, \ldots, \nu_{n, m}\right]^{T} } \\
\nu_{n, i}= & -K_{r-1, i}\left|\sigma_{i}^{\left(r_{i}-1\right)}\right|^{\alpha_{r_{i}, i}} \operatorname{sign}\left(\sigma_{i}^{\left(r_{i}-1\right)}\right)-\ldots \\
& -K_{0, i}\left|\sigma_{i}\right|^{\alpha_{1, i}} \operatorname{sign}\left(\sigma_{i}\right), \quad i=1, \ldots, m
\end{aligned}
$$

where $\boldsymbol{K}_{r-1}=\operatorname{diag}\left\{K_{r-1, i}\right\}, \ldots, \boldsymbol{K}_{0}=\operatorname{diag}\left\{K_{0, i}\right\}$, and their elements are chosen such that the $\boldsymbol{r}^{\text {th }}$ - order polynomials $p^{r_{i}}+K_{r-1, i} p^{r_{i}-1}+K_{r-2, i} p^{r_{i}-2} \ldots+K_{0, i}$ are Hurwitz. The scalars $\alpha_{1, i}, \ldots, \alpha_{r_{i}, i}$ satisfy

$$
\alpha_{k-1, i}=\frac{\alpha_{k, i} \alpha_{k+1, i}}{2 \alpha_{k+1, i}-\alpha_{k, i}}, k=2, \ldots, r_{i}, i=1, \ldots, m
$$

with $\alpha_{r_{i}+1, i}=1, \alpha_{r_{i}, i}=\alpha_{i}$.

Proof: Using Definition 1, this proposition is essentially a multi-input/multi-output generalization of the Proposition 8.1 in [22].

Using Proposition 1, there exists $\epsilon_{i}^{\prime} \in(0,1), i=1, \ldots, m$, such that for every $\alpha_{i}^{\prime} \in\left(1-\epsilon_{i}^{\prime}, 1\right)$, the $\boldsymbol{r}+\mathbf{1}^{t h}$ - order sliding mode is established in finite time by

$$
\begin{aligned}
& \nu_{n}^{\prime}=\left[\nu_{n, 1}^{\prime}, \nu_{n, 2}^{\prime}, \ldots, \nu_{n, m}^{\prime}\right]^{T} \\
& \nu_{n, i}^{\prime}=-K_{r, i}^{\prime}\left|\sigma_{i}^{\left(r_{i}\right)}\right|^{\alpha_{r_{i}+1, i}^{\prime}} \operatorname{sign}\left(\sigma_{i}^{\left(r_{i}\right)}\right)-\ldots \\
& -K_{0, i}^{\prime}\left|\sigma_{i}\right|^{\alpha_{1, i}^{\prime} \operatorname{sign}\left(\sigma_{i}\right), i=1, \ldots, m}
\end{aligned}
$$

The gain choices are the same as in Eq. (18). The scalars $\alpha_{1, i}^{\prime}, \ldots, \alpha_{r_{i}+1, i}^{\prime}$ satisfy

$$
\alpha_{k-1, i}^{\prime}=\frac{\alpha_{k, i}^{\prime} \alpha_{k+1, i}^{\prime}}{2 \alpha_{k+1, i}^{\prime}-\alpha_{k, i}^{\prime}}, k=2, \ldots, r_{i}+1, i=1, \ldots, m
$$

with $\alpha_{r_{i}+2, i}^{\prime}=1, \alpha_{r_{i}+1, i}^{\prime}=\alpha_{i}^{\prime}$.

Comparing Eq. (19) with Eq. (21), the nominal control part $\overline{\mathcal{B}}^{-1}\left(\boldsymbol{x}_{0}\right)\left(\boldsymbol{\nu}_{n}+\boldsymbol{y}_{c}^{(\boldsymbol{r})}-\boldsymbol{y}_{0}^{(\boldsymbol{r})}\right)$ in Eq. (8) approximately equals the nominal control increment $\overline{\mathcal{B}}^{-1}(\boldsymbol{x}) \boldsymbol{\nu}_{n}^{\prime} \Delta t$ in Eq. (16) if

1) $\overline{\mathcal{B}}^{-1}\left(\boldsymbol{x}_{0}\right) \approx \overline{\mathcal{B}}^{-1}(\boldsymbol{x})$

2) $\boldsymbol{y}^{(\boldsymbol{r})} \approx \boldsymbol{y}_{0}^{(\boldsymbol{r})}$

3) $\boldsymbol{K}_{r}^{\prime}=\frac{\boldsymbol{I}_{m}}{\Delta t}, \boldsymbol{K}_{r-1}^{\prime}=\frac{\boldsymbol{K}_{r-1}}{\Delta t}, \ldots, \boldsymbol{K}_{0}^{\prime}=\frac{\boldsymbol{K}_{0}}{\Delta t}$.

4) $\alpha_{r_{i}, i}^{\prime}=\alpha_{r_{i}, i}, \ldots, \alpha_{1, i}^{\prime}=\alpha_{1, i}$.

5) $\epsilon_{i} \ll 1, \alpha_{r_{i}+1, i}^{\prime}=\frac{2 \alpha_{r_{i}, i}^{\prime}}{1+\alpha_{r_{i}, i}^{\prime}}$.

As discussed in subsection III-A.1, the first two conditions can be satisfied using sufficiently small $\Delta t$. If the third and fourth conditions are satisfied, $\nu_{n, i}$ in Eq. (19) exactly equals $\nu_{n, i}^{\prime}+K_{r, i}^{\prime}\left|\sigma_{i}^{\left(r_{i}\right)}\right|^{\alpha_{r_{i}+1, i}^{\prime}} \operatorname{sign}\left(\sigma_{i}^{\left(r_{i}\right)}\right)$ in Eq. (21) multiplied with $\Delta t$. If $\alpha_{r_{i}+1, i}^{\prime}=1$, then $\left|\sigma_{i}^{\left(r_{i}\right)}\right|^{\alpha_{r_{i}+1, i}^{\prime}} \operatorname{sign}\left(\sigma_{i}^{\left(r_{i}\right)}\right)=$ $\sigma_{i}^{\left(r_{i}\right)}=y_{i}^{\left(r_{i}\right)}-y_{c_{i}}^{\left(r_{i}\right)}$, which means the first terms in Eq. (21), multiplied with $\Delta t$, approximate $\boldsymbol{y}_{c}^{(\boldsymbol{r})}-\boldsymbol{y}_{0}^{(\boldsymbol{r})}$ in Eq. (8), under the second and third conditions. In order to enforce the finite time convergence, the fifth condition is proposed, in which $\alpha_{r_{i}+1, i}^{\prime}$ satisfies Eq. (22), while $\alpha_{r_{i}+1, i}^{\prime}$ and $\alpha_{r_{i}, i}^{\prime}=\alpha_{r_{i}, i}$ are sufficiently close to one by requiring $\epsilon_{i} \ll 1$.

Remark 2: The unperturbed integral chains can also be stabilized in fixed-time using continuous $\boldsymbol{\nu}_{n}$ and $\boldsymbol{\nu}_{n}^{\prime}$ [25]. Analogous conditions can be derived which make $\overline{\mathcal{B}}^{-1}\left(\boldsymbol{x}_{0}\right)\left(\boldsymbol{\nu}_{n}+\boldsymbol{y}_{c}^{(\boldsymbol{r})}-\boldsymbol{y}_{0}^{(\boldsymbol{r})}\right)$ in Eq. (8) and $\overline{\mathcal{B}}^{-1}(\boldsymbol{x}) \boldsymbol{\nu}_{n}^{\prime} \Delta t$ in Eq. (16) approximately equal. 


\section{B. Comparisons of the Perturbation Terms}

$\varepsilon_{\text {ndi-s }}$ (Eq. (6)) and $\varepsilon_{\text {indi-s }}$ (Eq. (10)) have been compared in [8]. The main conclusions are summarized as follows:

1) A diagonally dominant structure of $\mathcal{B} \overline{\mathcal{B}}^{-1}$ and a sufficiently small $\Delta t$ ensure the boundedness of $\varepsilon_{\text {indi-s }}$, while $\varepsilon_{\text {ndi-s }}$ can become unbounded under the same conditions.

2) Even if $\varepsilon_{\text {ndi-s }}$ is bounded, under the same perturbation circumstance, there exists a $\Delta t$ such that the bound of $\varepsilon_{\text {indi-s }}$ is smaller than that of $\varepsilon_{\text {ndi-s }}$.

3) $\varepsilon_{\text {indi-s }}$ has less variations in different fault scenarios, while $\varepsilon_{\text {ndi-s }}$ is more fault-case dependent.

By virtue of these properties, the INDI-SMC framework is able to passively resist a wide range of uncertainties with lower sliding mode control/observer gains, as compared to the widely used NDI-SMC framework.

The properties of $\varepsilon_{\text {ho-s }}$ will be addressed here. Using Eq. (14), the first-order approximation of $\varepsilon_{\text {ho-s }}$ equals

$\varepsilon_{\mathrm{ho}-s} \approx \frac{\partial\left(\boldsymbol{\alpha}(\boldsymbol{x})+\mathcal{B}(\boldsymbol{x}) \boldsymbol{u}_{\mathrm{ho}-s}\right)}{\partial \boldsymbol{x}} \frac{\Delta \boldsymbol{x}}{\Delta t}+(\mathcal{B}-\overline{\mathcal{B}}) \frac{\Delta \boldsymbol{u}_{\mathrm{ho}-s}}{\Delta t}+\frac{\Delta \boldsymbol{d}}{\Delta t}$

Therefore, in comparison with Eq. (10), $\varepsilon_{\text {ho-s }}(t) \Delta t \approx$ $\varepsilon_{\text {indi-s }}(t), \dot{\varepsilon}_{\text {ho-s }}(t) \Delta t \approx \dot{\varepsilon}_{\text {indi-s }}(t)$ if $\boldsymbol{u}_{\text {ho-s }}(t) \approx \boldsymbol{u}_{\text {indi-s }}(t)$. Although $\boldsymbol{u}_{\text {ho-s }}$ and $\boldsymbol{u}_{\text {indi-s }}$ are designed using different control structures, their nominal increments are approximately equivalent if the conditions in III-A are satisfied. Moreover, basedon the equivalent control concept [2], [26], the required average control to maintain the sliding mode is identical in spite of the control structures. To be specific, once the $\boldsymbol{r}+\mathbf{1}^{\text {th }}$-order sliding mode is achieved by HOSMC, $\boldsymbol{\sigma}^{\boldsymbol{r}} \equiv \mathbf{0}$, thus $\boldsymbol{u}_{\text {ho- } s} \equiv \mathcal{B}^{-1}\left(\boldsymbol{y}_{c}^{(\boldsymbol{r})}-\boldsymbol{\alpha}-\boldsymbol{d}\right)$ according to Eq. (2). This value is also what $\boldsymbol{u}_{\text {indi- } s}$ must take on average to maintain the $\boldsymbol{r}^{\text {th }}$-order sliding mode [26]. In other words, once the $\boldsymbol{r}^{\text {th }}$ and $\boldsymbol{r}+\mathbf{1}^{t h}$-order sliding modes are respectively achieved by INDI-SMC and HOSMC, the condition $\boldsymbol{u}_{\text {ho-s }} \approx \boldsymbol{u}_{\text {indi-s }}$ is satisfied.

\section{Perturbation Compensations and the Minimum Possible Gains}

The three control structures discussed in Sec. II result in different closed-loop perturbation terms, which have different properties (III-B). It will be shown in this subsection that the minimum possible gains in $\boldsymbol{\nu}_{s}$ and $\boldsymbol{\nu}_{s}^{\prime}$ are determined by the norms of these perturbation terms.

1) First-Order Perturbation Compensation: For stabilizing the perturbed dynamics in Eqs. $(5,9)$, design an auxiliary sliding variable as $s=\sigma^{(\boldsymbol{r}-1)}-\int \boldsymbol{\nu}_{n}$, then $\dot{s}=\boldsymbol{\nu}_{s}+$ $\varepsilon_{\text {ndi-s/indi-s }}$ under the control of NDI and INDI based SMC. These dynamics can be stabilized using a first-order SMC as $\boldsymbol{\nu}_{s}=-\boldsymbol{K}_{s} \operatorname{sign}(\boldsymbol{s})=-\left[K_{s, 1} \operatorname{sign}\left(s_{1}\right), \ldots, K_{s, m} \operatorname{sign}\left(s_{m}\right)\right]^{T}$. The sufficient condition to enforce a sliding motion in finite time is that $K_{s, i}(t)>\eta+\left|\varepsilon_{\text {ndi-s } / \text { indi- } s, i}(t)\right|$, which coincides with the time-varying minimum possible gain requirement in [1]. $\eta$ is a small positive design constant for satisfying the $\eta$-reachability condition [1], [26].

When the sliding surface $s=0$ is reached, the equivalent closed-loop dynamics [18], [26] become $\boldsymbol{\sigma}^{(\boldsymbol{r})}=\boldsymbol{\nu}_{n}$, which recover the nominal condition despite the presence of perturbations. Using the $\boldsymbol{\nu}_{n}$ designed in III-A, the $\boldsymbol{r}^{t h}$-order sliding mode with respect to $\sigma$ is achieved.

Analogously, for HOSMC with artificially increased $r$, design an auxiliary sliding variable as $\boldsymbol{s}^{\prime}=\boldsymbol{\sigma}^{(\boldsymbol{r})}-\int \boldsymbol{\nu}_{n}^{\prime}$, then $\dot{s}^{\prime}=\nu_{s}^{\prime}+\varepsilon_{\text {ho-s }}$ using Eq. (13). $\boldsymbol{s}^{\prime}$ can be stabilized at zero in finite time by $\boldsymbol{\nu}_{s}^{\prime}=-\boldsymbol{K}_{s}^{\prime} \operatorname{sign}\left(\boldsymbol{s}^{\prime}\right)=$ $-\left[K_{s, 1}^{\prime} \operatorname{sign}\left(s_{1}^{\prime}\right), \ldots, K_{s, m}^{\prime} \operatorname{sign}\left(s_{m}^{\prime}\right)\right]^{T}$, with $K_{s, i}^{\prime}(t)>\eta+$ $\left|\varepsilon_{\text {ho-s }, i}(t)\right|$ for all $t$. As a result, within finite time, $\boldsymbol{\sigma}^{(\boldsymbol{r}+\mathbf{1})}=$ $\boldsymbol{\nu}_{n}^{\prime}$ on the sliding surface $\boldsymbol{s}^{\prime}=\mathbf{0}$. Using $\boldsymbol{\nu}_{n}^{\prime}$ designed in III-A ensures the establishment of the $\boldsymbol{r}+\mathbf{1}^{t h}$-order sliding mode with respect to $\sigma$.

Since there exists a $\Delta t$, such that the bound of $\varepsilon_{\text {indi-s }}$ is smaller than that of $\varepsilon_{\text {ndi-s }}$ (III-B), the minimum possible gains needed by INDI-SMC are lower than those required by NDI-SMC. Moreover, it has been shown in III-B that once the sliding modes are achieved, $\varepsilon_{\text {ho-s }}(t) \Delta t \approx \varepsilon_{\text {indi-s }}(t)$. Therefore, for maintaining the sliding modes, the minimum possible value of $\boldsymbol{K}_{s}^{\prime}(t)$ approximately equals $\boldsymbol{K}_{s}(t)$ used by INDI-SMC divided by $\Delta t$.

Remark 3: Although $\boldsymbol{K}_{s}^{\prime}(t)$ used by HOSMC with artificially increased $\boldsymbol{r}$ should be several orders of magnitude higher than $\boldsymbol{K}_{s}(t)$ used by INDI-SMC, the chattering magnitudes of these two methods are comparable since $\boldsymbol{\nu}_{s}^{\prime}$ is multiplied with $\Delta t$ in $\Delta \boldsymbol{u}_{\text {ho-s }}$ (Eq. (16)).

2) Super-twisting Observer: In this subsection, $\nu_{s}$ and $\nu_{s}^{\prime}$ will be designed by a super-twisting observer. The classical fixed-gain super-twisting observer will be designed first, then the observer with time-varying gain will be discussed.

Assumption 2: The time derivatives of $\varepsilon_{\text {ndi-s }}(t)$ (Eq. (6)), $\varepsilon_{\text {indi-s }}(t)\left(\right.$ Eq. (10)), $\varepsilon_{\text {ho-s }}(t)$ (Eq. (14)) are bounded. Denote their upper-bounds as: $\left|\dot{\varepsilon}_{\text {ndi-s }, i}(t)\right|<\bar{\varepsilon}_{\text {ndi-s }, i},\left|\dot{\varepsilon}_{\text {indi- } s, i}(t)\right|<$ $\bar{\varepsilon}_{\text {indi- } s, i},\left|\dot{\varepsilon}_{\text {ho- } s, i}(t)\right|<\bar{\varepsilon}_{\text {ho- } s, i}$.

Use the same auxiliary sliding variables $s$ and $s^{\prime}$ as in IIIC.1, and design

$$
\boldsymbol{\nu}_{s}=-\boldsymbol{\lambda}|\boldsymbol{s}|^{1 / 2} \operatorname{sign}(s)-\boldsymbol{\beta} \int \operatorname{sign}(s)
$$

where $\boldsymbol{\lambda}=\operatorname{diag}\left\{\lambda_{i}\right\}, \quad \lambda_{i}=1.5 \bar{\varepsilon}_{\text {ndi-s/indi-s,i }}^{1 / 2}, \quad \boldsymbol{\beta}=$ $\operatorname{diag}\left\{\beta_{i}\right\}, \quad \beta_{i}=1.1 \bar{\varepsilon}_{\text {ndi-s } / \text { indi- } s}, \quad i=1, \ldots, m$, then $s=\dot{s}=$ $\mathbf{0}$ is established in finite time [4]. On the sliding surfaces, $\nu_{s}(t)$ observes $-\varepsilon_{\text {ndi-s/indi-s }}(t)$ according to Eqs. $(5,9)$, thus the unperturbed dynamics $\boldsymbol{\sigma}^{(\boldsymbol{r})}=\boldsymbol{\nu}_{n}$ are recovered in finite time. Analogously, $s^{\prime}$ is stabilized in finite time by

$$
\nu_{s}^{\prime}=-\boldsymbol{\lambda}^{\prime}\left|\boldsymbol{s}^{\prime}\right|^{1 / 2} \operatorname{sign}\left(\boldsymbol{s}^{\prime}\right)-\boldsymbol{\beta}^{\prime} \int \operatorname{sign}\left(\boldsymbol{s}^{\prime}\right)
$$

where $\boldsymbol{\lambda}^{\prime}=\operatorname{diag}\left\{\lambda_{i}^{\prime}\right\}, \quad \lambda_{i}^{\prime}=1.5 \bar{\varepsilon}_{\text {ho-s,i }}^{1 / 2}, \boldsymbol{\beta}=\operatorname{diag}\left\{\beta_{i}\right\}, \beta_{i}=$ $1.1 \bar{\varepsilon}_{\text {ho-s }, i}, \quad i=1, \ldots, m$, then $\nu_{s}^{\prime}$ observes $-\varepsilon_{\text {ho- } s}$ in finite time. Consequently, the unperturbed dynamics $\boldsymbol{\sigma}^{(\boldsymbol{r}+\mathbf{1})}=\boldsymbol{\nu}_{n}^{\prime}$ are achieved in finite time. Adopting $\boldsymbol{\nu}_{n}^{\prime}$ designed in III-A enforces the $\boldsymbol{r}+\mathbf{1}^{\text {th }}$-order sliding mode with respect to $\sigma$.

Once the sliding modes are achieved $\dot{\varepsilon}_{\text {ho-s }}(t) \Delta t \approx$ $\dot{\varepsilon}_{\text {indi-s }}(t)$ according to the analyses in III-B. Therefore, in view of the gain conditions, $\boldsymbol{\lambda}^{\prime}$ approximately equals $\boldsymbol{\lambda}$ used by INDI-SMC divided by $\sqrt{\Delta t}$ to maintain the sliding mode. 
Moreover, $\boldsymbol{\beta}^{\prime}$ approximately equals $\boldsymbol{\beta}$ used by INDI-SMC divided by $\Delta t$.

The super-twisting observers designed by Eqs. $(24,25)$ use fixed gain matrices. Although the discontinuous signum functions have been integrated, the chattering effects can not be totally eliminated [1], [2]. This issue inspires some recent researches to use time-varying $\boldsymbol{\beta}(t)$ and $\boldsymbol{\beta}^{\prime}(t)$ for mitigating the chattering effects. Different adaptation mechanisms are proposed in [1], [2], [3] which make $\beta_{i}(t)$ and $\beta_{i}^{\prime}(t)$ as low as possible, whilst still guaranteeing that $\beta_{i}(t)>$ $\left|\dot{\varepsilon}_{\text {ndi- } s / \text { indi- } s, i}(t)\right|, \beta_{i}^{\prime}(t)>\left|\dot{\varepsilon}_{\text {ho- } s, i}(t)\right|, i=1, \ldots, m$. In view of these conditions, for maintaining the sliding modes, the minimum possible value of $\boldsymbol{\beta}^{\prime}(t)$ still approximately equals $\boldsymbol{\beta}(t)$ used by INDI-SMC divided by $\Delta t$.

Remark 4: When the super-twisting observers are used, the chattering magnitude of HOSMC with artificially increased $\boldsymbol{r}$ is similar with that of INDI-SMC, because $\boldsymbol{\nu}_{s}^{\prime}$ will be multiplied with $\Delta t$ in Eq. (16).

Remark 5: The influences of control structures on the minimum possible control/observer gain values are not restricted to the two preceding $\boldsymbol{\nu}_{s} / \boldsymbol{\nu}_{s}^{\prime}$ designs. This is because for most sliding mode control/observer designs, the minimum possible gains are positively correlated with the norms of the closed-loop uncertainties or the norms of the corresponding derivatives.

\section{NumericAl COMPARISONS}

Consider a nonlinear control-affine system:

$$
\ddot{x}=\dot{x}+3 x+x^{2}+10 u+d
$$

where the external disturbance $d=0.5 \sin (10 t)$. The control aim is making the output $y=x$ track the reference signal $y_{c}=5 \sin (t)$ in finite time. The relative degree $r$ of the system equals two. As compared to Eq. (2), $\alpha=\dot{x}+3 x+x^{2}$, $\mathcal{B}=10$ for this system. Assume the estimated model used for control design is $\overline{\boldsymbol{\alpha}}=x+0.5 x^{2}, \overline{\mathcal{B}}=15$, which has mismatches with the plant. The disturbance $d$ is unknown by the controllers. Design the sliding variable as $\sigma=y-y_{c}$. The initial conditions are: $y(t=0)=2, \dot{y}(t=0)=0$. The sampling frequency is $f_{s}=1000 \mathrm{~Hz}(\Delta t=0.001 \mathrm{~s})$.

Using Eqs. $(4,8,12)$, the control inputs using the three discussed control structures are

$$
\begin{aligned}
u_{\text {ndi-s }} & =(1 / 15)\left(\nu_{n}+\nu_{s}+\ddot{y}_{c}-\left(x+0.5 x^{2}\right)\right) \\
\Delta u_{\text {indi-s }} & =(1 / 15)\left(\nu_{n}+\nu_{s}+\ddot{y}_{c}-\ddot{y}_{0}\right) \\
\dot{u}_{\text {ho-s }} & =(1 / 15)\left(\nu_{n}^{\prime}+\nu_{s}^{\prime}+\dddot{y}_{c}\right)
\end{aligned}
$$

$\nu_{n}$ in Eqs. $(27,28)$ are designed using Proposition 1 with the control parameters $K_{0}=16, K_{1}=6.4, \alpha_{2}=0.98$. $\alpha_{1}$ is calculated using Eq. (20) as $\alpha_{1}=0.96$. Design $\nu_{n}^{\prime}$ using Eq. $(21,22)$, while satisfying the five conditions in subsection III-A.2, then the nominal control increment $\overline{\mathcal{B}}^{-1}(\boldsymbol{x}) \boldsymbol{\nu}_{n}^{\prime} \Delta t$ in Eq. (16) becomes close to the nominal control increment of INDI-SMC (Eq. (8)). Explicitly, $K_{0}^{\prime}=$ $16 / \Delta t, K_{1}^{\prime}=6.4 / \Delta t, K_{2}^{\prime}=1 / \Delta t, \alpha_{2}^{\prime}=0.98, \alpha_{1}^{\prime}=$ $0.96, \alpha_{3}^{\prime}=0.99 . \nu_{s}$ and $\nu_{s}^{\prime}$ are designed using the supertwisting observer in III-C.2, their gain selection issues will be addressed later on.

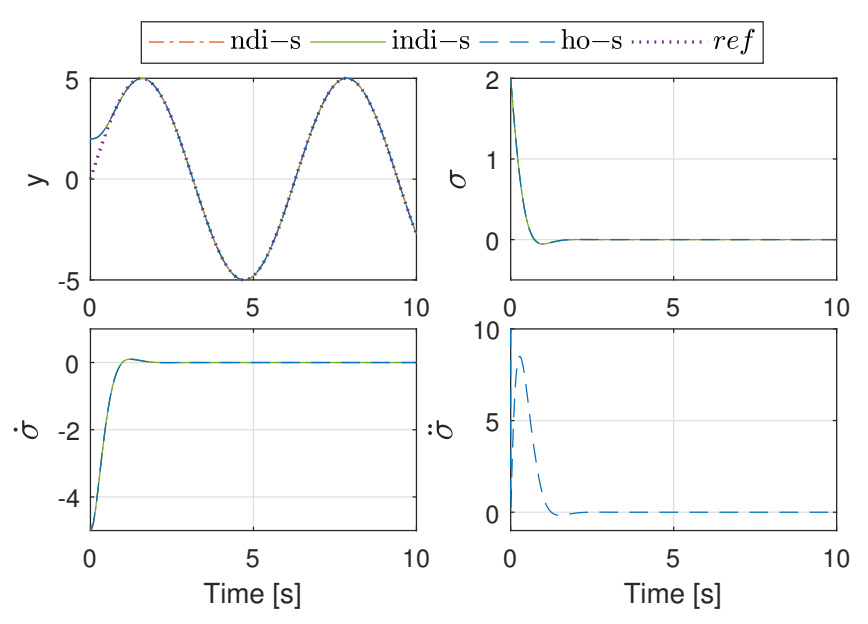

Fig. 1. Evolutions of the output $y$, and the derivatives of $\sigma$.

Evolutions of the output, and the derivatives of $\sigma$ using three different control structures are shown in Fig. 1. It can be seen that the $2^{n d}$-order sliding mode with respect to $\sigma$ is achieved within finite time by both NDI-SMC and INDISMC despite the presence of the disturbance and model mismatches. The $3^{\text {rd }}$-order sliding mode is also established by HOSMC within finite time. Because the control parameters are chosen correspondingly, similar transient responses are present, with $\left|\sigma_{\text {ndi-s }}-\sigma_{\text {indi-s }}\right|<0.020$ and $\left|\sigma_{\text {indi-s }}-\sigma_{\text {ho-s }}\right|<$ 0.007 throughout the entire time history.

As discussed in subsection III-B, the three considered control structures result in different perturbation terms. The evolutions of these terms are shown in the left subplot of Fig. 2, from which it can be seen that $\varepsilon_{\text {ndi-s }}$ (Eq. (6)) is quite different form $\varepsilon_{\text {indi-s }} / \Delta t$ (Eq. (10)) and $\varepsilon_{\text {ho-s }}$ (Eq. (14)). This difference can be revealed by comparing the formulations of the uncertainty terms, where it can be found that only $\varepsilon_{\text {ndi-s }}$ contains $\boldsymbol{\alpha}-\overline{\boldsymbol{\alpha}}$. Worse $\overline{\boldsymbol{\alpha}}$ estimation would result in increased $\left|\varepsilon_{\text {ndi-s }}\right|$. The upper bound of $\varepsilon_{\text {indi-s } s}$ is three orders of magnitude smaller than that of $\varepsilon_{\text {ndi-s }}$ and $\varepsilon_{\text {ho-s }}$. Except for rare time instances where $\varepsilon_{\text {ndi-s }}=0$, while $\varepsilon_{\text {indi-s }} \neq 0,\left|\varepsilon_{\text {indi-s }}(t)\right|<\left|\varepsilon_{\text {ndi-s }}(t)\right|$ throughout the entire time history. This inequality is beneficial for chattering reduction, since the time-varying minimum possible switching gain is determined by the absolute value of the uncertainty (III-C.1).

Moreover, for all $t \in(0,10],\left|\varepsilon_{\text {indi-s }} / \Delta t-\varepsilon_{\text {ho-s }}\right|<$ 1.24 , which verifies the analyses in subsection III-B. As a consequence, $K_{s}^{\prime}(t)$ used by HOSMC should be three orders of magnitude higher than $K_{s}(t)$ used by INDI-SMC if $\nu_{s}$ and $\nu_{s}^{\prime}$ are designed using the first-order SMC (III-C.1). Nevertheless, these two methods will lead to comparable chattering magnitude according to Remark 3.

The evolutions of the uncertainty derivatives are shown in the right subplot of Fig. 2. $\dot{\varepsilon}_{\text {ndi-s }}$ has quite different responses as compared to $\dot{\varepsilon}_{\text {indi-s }} / \Delta t$ and $\dot{\varepsilon}_{\text {ho- } s}$. For all $t \in(0,10]$, $\left|\dot{\varepsilon}_{\text {indi- } s} / \Delta t-\dot{\varepsilon}_{\text {ho- } s}\right|<2.51$, which verifies the analyses in subsection III-B. In this simulation case, $\bar{\varepsilon}_{\text {ndi-s }}=120, \bar{\varepsilon}_{\text {ho-s }}=$ 350 , while $\bar{\varepsilon}_{\text {indi-s }}$ is only 0.35 . Using the gain conditions imposed on Eqs. $(24,25)$, the super-twisting observations are 

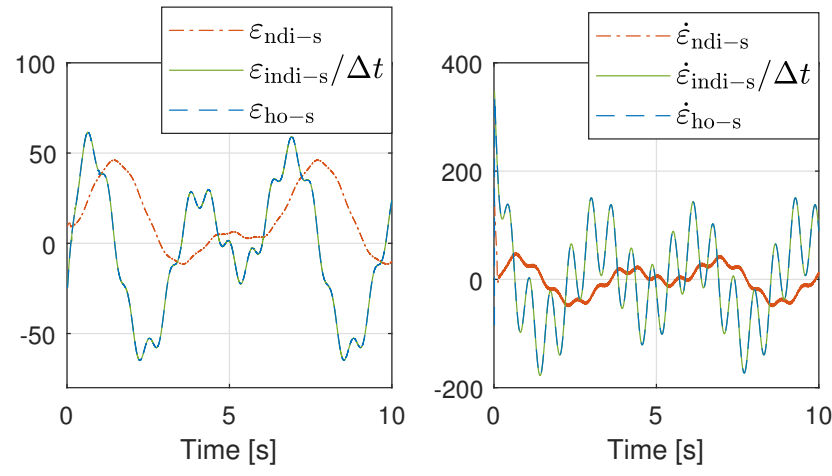

Fig. 2. Evolutions of the uncertainties (left) and their derivatives (right).

illustrated in Fig. 3. All the three observers converge within 0.3 seconds, after which the nominal dynamics are recovered, thus the higher-order sliding modes are established in spite of perturbations as shown in Fig. 1.

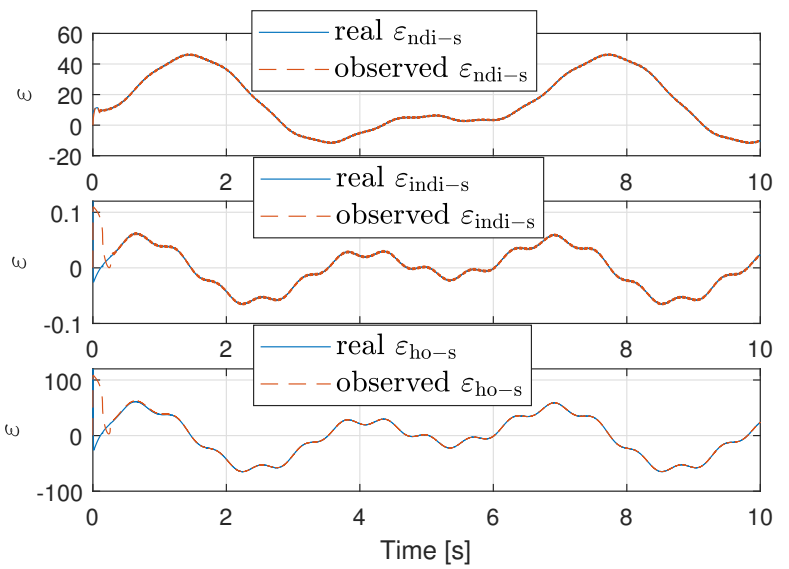

Fig. 3. The real and observed (via super-twisting observers) uncertainties.

Even though the observations provided by the supertwisting observers are continuous, chattering is only attenuated instead of being totally rejected by the integration in Eqs. $(24,25)$ [1]. This is verified by Fig. 4, where the NDI-SMC control input has a chattering magnitude of about 0.06 . By contrast, because of the three orders of magnitude smaller observer gains, $u_{\text {indi-s }}$ is much smoother, which is verified by Fig. 4 in both time and frequency domains. The right subplot of Fig. 4 illustrates the Welch's power spectral density estimation of the control inputs (using the Matlab command "pwelch"). It can also be observed from Fig. 4 that although HOSMC has even higher observer gains than NDI-SMC, the chattering magnitude of $u_{\text {ho-s }}$ is comparable with that of $u_{\text {indi-s } s}$. This has been explained by Remark 4 and is verified by Fig. 4. Furthermore, because the five conditions in subsection III-A.2 are satisfied, HOSMC with artificially increased $r$ and INDI-SMC provide similar control inputs as $\left|u_{\text {indi-s }}-u_{\text {ho-s }}\right|<0.014$ for all $t \in(0,10] \mathrm{s}$.

It can be seen from the evolutions of the uncertainty derivatives that once the sliding modes are established, the gains determined by the upper bounds can be conserva- tive. As argued in subsection III-A.2, even if the observer gains are adapted, their minimum possible values are still constrained by the norms of the uncertainty derivatives. In view of the right subplot of Fig. 2, INDI-SMC has the smallest minimum possible observer gains among all the three discussed control structures.

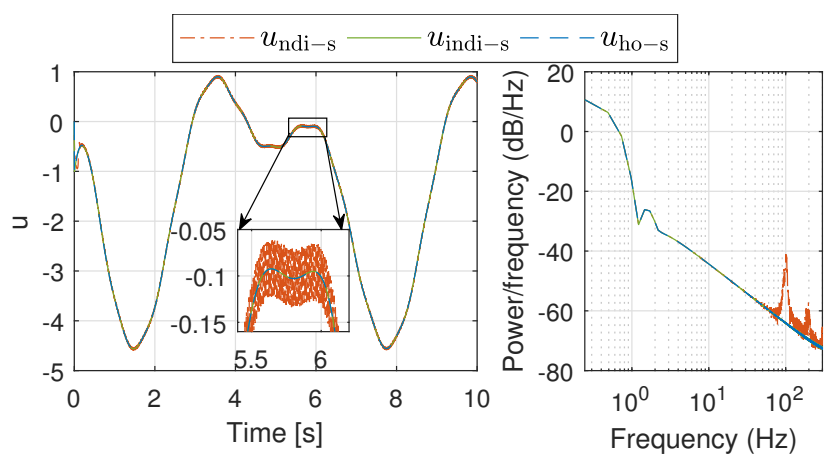

Fig. 4. Control inputs in the time (left) and the frequency (right) domains.

In view of the preceding results, when the five conditions in III-A.2 are satisfied, INDI-SMC and HOSMC with artificially increased $r$ have similar performance and chattering magnitude. Their resulting uncertainties, uncertainty derivatives, and the minimum possible gains are directly connected by $\Delta t$. A natural research question would be: what if the conditions in III-A.2 are unsatisfied? This is tested by a case where the previously used $K_{0}^{\prime}$ and $K_{1}^{\prime}$ are multiplied with 0.3, with the results shown in Fig. 5.

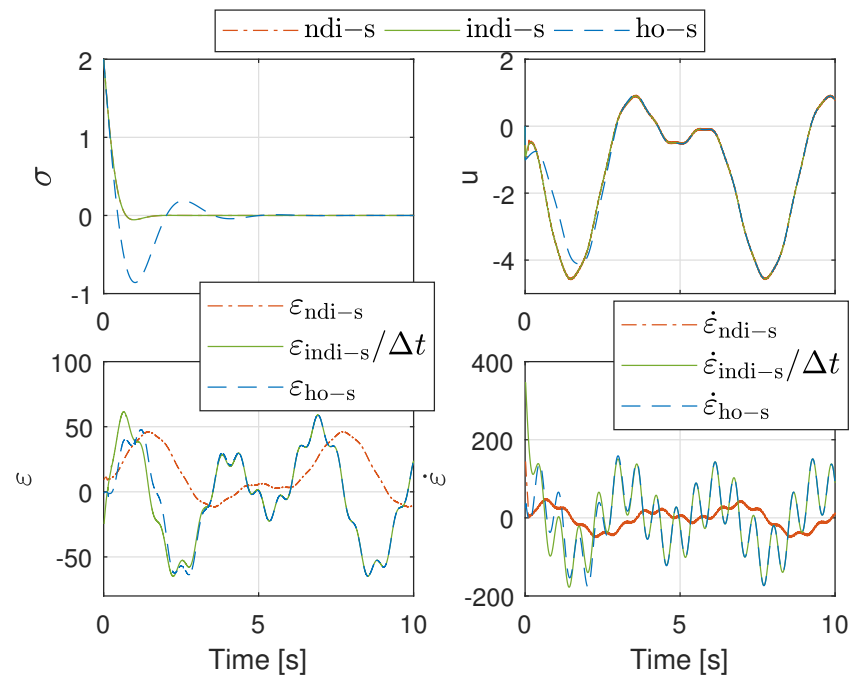

Fig. 5. A test case in which the conditions in III-A.2 are not satisfied.

In this case, Fig. 5 shows that the transient responses of INDI-SMC and HOSMC no long resemble each other. Since $K_{0}^{\prime}$ and $K_{1}^{\prime}$ are reduced, it takes longer (six seconds) for HOSMC to converge. However, once the sliding modes are enforced, $\boldsymbol{u}_{\text {ho-s }} \approx \boldsymbol{u}_{\text {indi- } s}$. Furthermore, $\varepsilon_{\text {ho-s }}(t) \approx$ $\varepsilon_{\text {indi-s }}(t) / \Delta t, \dot{\varepsilon}_{\text {ho-s }}(t) \approx \dot{\varepsilon}_{\text {indi-s }}(t) / \Delta t$ after $t=6 \mathrm{~s}$ in Fig. 5 . These results verify the analyses in subsection III-B. 


\section{COnClusions}

In this paper, three control structures for inducing higherorder sliding modes: nonlinear dynamic inversion based sliding mode control (abbreviated to NDI-SMC, which designs $\boldsymbol{u}_{\text {ndi-s }}$ ), incremental sliding mode control (indicated as INDISMC, which designs $\left.\Delta \boldsymbol{u}_{\text {indi-s }}\right)$, and higher-order sliding mode control (HOSMC) with artificially increased relative degree by one order (designs $\dot{\boldsymbol{u}}_{\text {ho-s }}$ ) are compared analytically and numerically. $\boldsymbol{u}_{\text {ndi-s }}$ needs both the estimations of system dynamics and control effectiveness for control implementation, while only the estimated control effectiveness is needed by $\Delta \boldsymbol{u}_{\text {indi-s }}$ and $\dot{\boldsymbol{u}}_{\text {ho-s }}$. Nevertheless, $\Delta \boldsymbol{u}_{\text {indi-s }}$ depends on the measurements/estimations of the output derivatives, while $\dot{\boldsymbol{u}}_{\text {ho-s }}$ relies on the numerically differentiated output derivatives and the higher-order derivatives of the tracking commands.

Although the considered HOSMC and INDI-SMC originate from completely different ideas, their nominal control increments are found to be approximately equivalent if the sampling interval $\Delta t$ is sufficiently small and if their control parameters satisfy the conditions presented in subsection III-A. When these conditions are satisfied, similar transient responses and control performance are achieved by HOSMC and INDI-SMC. Otherwise, although their transient responses would be different, once the higher-order sliding modes are achieved, the control efforts needed to maintain the sliding modes become the same.

In the presence of external disturbances and model uncertainties, these three control structures result in different closed-loop uncertainty terms, namely $\varepsilon_{\text {ndi- } s}, \varepsilon_{\text {indi- } s}$ and $\varepsilon_{\text {ho- } s}$. It is verified in this paper that there exists a $\Delta t$ such that $\left\|\varepsilon_{\text {indi-s }}(t)\right\|<\left\|\varepsilon_{\text {ndi-s }}(t)\right\|$ almost everywhere. Moreover, it is found that once the sliding modes are enforced, $\varepsilon_{\text {ho- } s}(t) \Delta t \approx$ $\varepsilon_{\text {indi-s }}(t), \dot{\varepsilon}_{\text {ho- } s}(t) \Delta t \approx \dot{\varepsilon}_{\text {indi-s }}(t)$. For most sliding mode control/observer designs, the minimum possible gains are determined by the norms of the uncertainty terms or the norms of the corresponding derivatives. Therefore, for maintaining the sliding modes, the minimum possible gains required by HOSMC approximately equal those needed by INDI-SMC divided by $\Delta t$. Even so, these two control structures lead to comparable chattering magnitudes, which are efficaciously reduced as compared to NDI-SMC.

\section{REFERENCES}

[1] C. Edwards and Y. B. Shtessel, "Adaptive continuous higher order sliding mode control," Automatica, vol. 65, no. March, pp. 183-190, mar 2016

[2] V. I. Utkin and A. S. Poznyak, "Adaptive sliding mode control with application to super-twist algorithm: Equivalent control method," Automatica, vol. 49, no. 1, pp. 39-47, 2013.

[3] C. Edwards and Y. Shtessel, "Adaptive dual-layer super-twisting control and observation," International Journal of Control, vol. 89, no. 9, pp. 1759-1766, 2016.

[4] C. Edwards and Y. B. Shtessel, "Continuous higher order sliding mode control based on adaptive disturbance compensation," in 2014 13th International Workshop on Variable Structure Systems (VSS), vol. 47, no. 3. IEEE, jun 2014.

[5] X. Wang, E. Van Kampen, Q. P. Chu, and P. Lu, "Stability Analysis for Incremental Nonlinear Dynamic Inversion Control," Journal of Guidance, Control, and Dynamics, 2019. [Online]. Available: https://arc.aiaa.org/doi/10.2514/1.G003791
[6] S. Sieberling, Q. P. Chu, and J. A. Mulder, "Robust Flight Control Using Incremental Nonlinear Dynamic Inversion and Angular Acceleration Prediction," Journal of Guidance, Control, and Dynamics, vol. 33, no. 6, pp. 1732-1742, 2010.

[7] P. Lu, E. van Kampen, C. de Visser, and Q. Chu, "Aircraft fault-tolerant trajectory control using Incremental Nonlinear Dynamic Inversion," Control Engineering Practice, vol. 57, no. December, pp. 126-141, dec 2016.

[8] X. Wang, E. van Kampen, Q. Chu, and P. Lu, "Incremental SlidingMode Fault-Tolerant Flight Control," Journal of Guidance, Control, and Dynamics, vol. 42, no. 2, pp. 244-259, feb 2019.

[9] P. Smith, "A Simplified Approach to Nonlinear Dynamic Inversion Based Flight Control," in 23rd Atmospheric Flight Mechanics Conference. Reston, VA: AIAA, 1998.

[10] P. Simplício, M. Pavel, E. van Kampen, and Q. Chu, "An acceleration measurements-based approach for helicopter nonlinear flight contro using Incremental Nonlinear Dynamic Inversion," Control Engineering Practice, vol. 21, no. 8, pp. 1065-1077, aug 2013.

[11] X. Wang, E. Van Kampen, R. De Breuker, and Q. P. Chu, "Flexible Aircraft Gust Load Alleviation with Incremental Nonlinear Dynamic Inversion," Journal of Guidance,Control, and Dynamics, 2019. [Online]. Available: https://arc.aiaa.org/doi/10.2514/1.G003980

[12] S. Sun, L. Sijbers, X. Wang, and C. de Visser, "High-Speed Flight of Quadrotor Despite Loss of Single Rotor," IEEE Robotics and Automation Letters, vol. 3, no. 4, pp. 3201-3207, oct 2018

[13] F. Grondman, G. Looye, R. O. Kuchar, Q. P. Chu, and E. Van Kampen, "Design and Flight Testing of Incremental Nonlinear Dynamic Inversion-based Control Laws for a Passenger Aircraft," in 2018 AIAA Guidance, Navigation, and Control Conference, no. January. Kissimmee, Florida: AIAA, jan 2018.

[14] T. Keijzer, G. Looye, Q. P. Chu, and E. Van Kampen, "Design and Flight Testing of Incremental Backstepping based Control Laws with Angular Accelerometer Feedback," in AIAA Scitech 2019 Forum, no. January. San Diego, California: AIAA, jan 2019, pp. 1-25.

[15] Wu-Chung Su, S. Drakunov, and U. Ozguner, "An O(T/sup 2/) boundary layer in sliding mode for sampled-data systems," IEEE Transactions on Automatic Control, vol. 45, no. 3, pp. 482-485, mar 2000

[16] R. Morgan and U. Ozguner, "A decentralized variable structure control algorithm for robotic manipulators," IEEE Journal on Robotics and Automation, vol. 1, no. 1, pp. 57-65, 1985.

[17] A. Levant, "Higher-order sliding modes, differentiation and outputfeedback control," International Journal of Control, vol. 76, no. 9-10, pp. 924-941, 2003.

[18] M. Defoort, T. Floquet, A. Kokosy, and W. Perruquetti, "A novel higher order sliding mode control scheme," Systems and Control Letters, vol. 58, no. 2, pp. 102-108, 2009.

[19] A. Levant, "Sliding order and sliding accuracy in sliding mode control," International Journal of Control, vol. 58, no. 6, pp. 12471263, dec 1993.

[20] A. F. Filippov, Differential Equations with Discontinuous Righthand Sides, ser. Mathematics and Its Applications, F. M. Arscott, Ed. Dordrecht: Springer Netherlands, 1988, vol. 18.

[21] J. Wang, Q. Zong, R. Su, and B. Tian, "Continuous high order sliding mode controller design for a flexible air-breathing hypersonic vehicle," ISA Transactions, vol. 53, no. 3, pp. 690-698, 2014.

[22] S. P. Bhat and D. S. Bernstein, "Geometric homogeneity with applications to finite-time stability," Mathematics of Control, Signals, and Systems, vol. 17, no. 2, pp. 101-127, 2005.

[23] E. J. J. Smeur, Q. P. Chu, and G. C. H. E. de Croon, "Adaptive Incremental Nonlinear Dynamic Inversion for Attitude Control of Micro Air Vehicles," Journal of Guidance, Control, and Dynamics, vol. 39, no. 3, pp. 450-461, 2016.

[24] P. Yu, Y. Shtessel, and C. Edwards, "Continuous higher order sliding mode control with adaptation of air breathing hypersonic missile," International Journal of Adaptive Control and Signal Processing, vol. 30, no. 8-10, pp. 1099-1117, aug 2016.

[25] M. Basin, Y. Shtessel, and F. Aldukali, "Continuous finite- and fixedtime high-order regulators," Journal of the Franklin Institute, vol. 353 no. 18, pp. 5001-5012, 2016.

[26] V. Utkin, J. Guldner, and J. Shi, Sliding Mode Control in ElectroMechanical Systems, Second Edition, ser. Automation and Control Engineering. London: CRC Press, may 2009, vol. 31. 